Supporting Information I

\title{
Which micropollutants in water environments deserve more attention globally?
}

Yun Yang, ${ }^{+}$Xiangru Zhang, ${ }^{+,}$Jingyi Jiang, ${ }^{+}$Jiarui Han, ${ }^{+}$Wanxin $\mathrm{Li},{ }^{+}$Xiaoyan Li, ${ }^{\ddagger}$

Kenneth Mei Yee Leung, ${ }^{\S}$ Shane A. Snyder,$\varsigma$ and Pedro J.J. Alvarez, ${ }^{\perp}$

${ }^{+}$Department of Civil and Environmental Engineering, The Hong Kong University of Science and Technology, Clear Water Bay, Hong Kong, China

${ }^{\ddagger}$ Department of Civil Engineering, The University of Hong Kong, Pokfulam, Hong Kong, China

s State Key Laboratory of Marine Pollution and Department of Chemistry, City University of Hong Kong, Tat Chee Avenue, Kowloon, Hong Kong, China

$\zeta$ Nanyang Technological University, Nanyang Environment \& Water Research Institute, 1 Cleantech Loop, CleanTech One, \#06-08, 637141, Singapore

${ }^{\perp}$ Department of Civil and Environmental Engineering, Rice University, Houston, TX, 77005, United States

*Corresponding author.

E-mail address: xiangru@ust.hk (X. Zhang).

The supporting information consists of 21 pages, 1 text and 3 tables. 


\section{Contents}

\section{Occurrence and toxicity information of frequently detected MPs}

\section{References}

Table S1 - The concentrations, PNEC and ADI values of the commonly detected PPCPs in various water bodies.

Table S2 - The concentrations, PNEC and ADI values of the commonly detected pesticides in various water bodies.

Table S3 - The concentrations, PNEC and ADI values of the commonly detected industrial chemicals in various water bodies. 


\section{Text S1. Occurrence and toxicity information of frequently detected MPs}

\section{Pharmaceuticals and personal care products (PPCPs)}

A variety of chemicals can be classified as PPCPs based on their usage instead of chemical structure, thereby the behaviors of PPCPs in aquatic environments are greatly different. ${ }^{1}$ Pharmaceuticals include any chemicals used to treat diseases or accelerate growth of human and animals. Personal care products are one type of household products designed to improve life quality, such as shampoo, sunscreen, and insect repellents. Commonly detected PPCPs can be further divided into different subgroups according to therapeutic uses, such as antibiotics, nonsteroidal anti-inflammatory drugs (NSAIDs), and hormones. Many PPCP have been reported to be toxic to aquatic biotas or humans. ${ }^{2-3}$ In addition, endocrine disruption effects and increasing drug-resistant bacterial strain in aquatic environments are also related with the exposure of PPCPs. ${ }^{4}$ As can be seen in Table S1, typical occurrence levels of PPCPs vary substantially in different water compartments. PPCPs mainly present at $\mathrm{ng} / \mathrm{L}$ to low $\mu \mathrm{g} / \mathrm{L}$ in surface and coastal water. As for groundwater, the maximum concentrations of PPCPs are considerably larger reaching high $\mu \mathrm{g} / \mathrm{L}$ level, but the ranges are broader as well. Moreover, the properties of PPCPs from different subgroups are also substantially different. Antibiotics, lipid regulators, and several illicit drugs are more frequently detected, which is indicative of their extensive temporal and spatial impacts in aquatic environments. By contrast, hormones, carbamazepine, and diethyltoluamide (DEET) have lower PNEC or ADI values, signifying their high toxicity to environment and human health.

\subsection{Antibiotics}

Antibiotics are chemicals that can inhibit bacterial growth to prevent or treat bacterial infections. They are a group of frequently detected PPCPs in aquatic environments. ${ }^{5}$ For example, $\mathrm{Bu}$ et $\mathrm{al} .{ }^{6}$ reported the occurrence of 112 PPCPs in Eastern China, and over $75 \%$ of them were antibiotics. The major source of antibiotics is the veterinary use. ${ }^{7}$ Enormous usage of antibiotics, coupled with their robust resistance to biodegradation, contributes to their extensive occurrence in aquatic environments. ${ }^{8}$ Many antibiotics have been reported to exhibit toxic effects to a variety of 
aquatic biotas. ${ }^{9-11}$ A more significant issue may be the rampant increase of resistance in bacterial strains due to uncontrolled use of antibiotics, which may progressively affect organisms in higher trophic levels via food chains. ${ }^{2}$ According to Table S1, roxithromycin is the antibiotic with the highest occurrence concentration of $3.7 \mu \mathrm{g} / \mathrm{L}$. The lowest PNEC and ADI values are recorded for ofloxacin (16 ng/L) and clarithromycin $\left(2 \times 10^{-4} \mathrm{mg} / \mathrm{kg}\right.$-day $)$, respectively.

\subsection{Lipid regulators}

Lipid regulators are prescription drugs that are used to lower lipid (e.g., cholesterol) levels in the blood. They are ubiquitous in aquatic environments mainly because of their high daily dosage on humans. The removal efficiencies of lipid regulators in wastewater treatment plants (WWTPs) are also low, ranging from $6 \%$ to $50 \% .^{2}$ Clofibric acid was the first detected lipid regulator in wastewater effluents, ${ }^{12}$ which, coupled with fenofibric acid and gemfibrozil, is among the most commonly reported lipid regulators in aquatic environments. The maximum concentration of clofibric acid is $7.3 \mu \mathrm{g} / \mathrm{L}$ in groundwaters. The PNEC value of clofibric acid is also relatively high, indicating that its risk level on aquatic environments may be moderate. By contrast, gemfibrozil has much lower occurrence levels and PNEC value.

\subsection{Analgesics and NASIDs}

Analgesics are primarily used to relieve pain, and most analgesics also have anti-inflammatory effects, thus here analgesics and NASIDs are classified into the same subgroup of PPCPs. Acetaminophen is the most prevalently used analgesic and often sold in large amounts without prescription. The detection frequency of acetaminophen is relatively low, mainly because it can readily degrade and be effectively removed in WWTPs. ${ }^{13}$ However, the maximum concentration of acetaminophen was reported to be $10 \mu \mathrm{g} / \mathrm{L}$ in a nationwide reconnaissance in the United States. ${ }^{14}$ Salicylic acid mainly comes from the degradation of another popular analgesic, acetylsalicylic acid. Despite the massive input of salicylic acid, it can also be efficiently removed in WWTPs, leading to relatively low concentrations in receiving water bodies. ${ }^{15}$ Diclofenac and ibuprofen are two widely used NASIDs, and their annual consumptions in Germany were reported

to be 75 and 180 tons, respectively. ${ }^{16}$ However, diclofenac usually presents at a higher 
concentration than ibuprofen, because the former is more refractory in WWTPs. By contrast, ibuprofen degrades easily, although sporadic high occurrence of ibuprofen was also reported in groundwaters. The highest detection frequency of analgesics is recorded for naproxen, but the occurrence levels of naproxen in aquatic environments are relatively low.

\subsection{Hormones}

Hormones are important for regulating physiological activities, and steroid estrogens are biologically active hormones that are of environmental relevance. Three natural steroid estrogens (E1, E2 and E3) are mainly released from human and livestock, while a synthesis estrogen (EE2) is widely prescribed as a contraceptive. ${ }^{17-18}$ Steroid estrogens have low solubility in water and tend to be adsorbed onto sediments due to their high $\mathrm{K}_{\mathrm{ow}}$ values. ${ }^{19}$ Table $\mathrm{S} 1$ shows that all four steroid estrogens basically present at ng/L levels in aquatic environments, and their PNEC and ADI values are considerably lower than those of other PPCPs. EE2 has the lowest PNEC value $(0.035 \mathrm{ng} / \mathrm{L})$ among four hormones. The low hazard parameters of hormones might be because the endpoint for PNEC and ADI values is endocrine disrupting effect rather than apical toxic effects (e.g., survival), and steroid estrogens were reported to be the primary contributors on the estrogenic activity in aquatic environments. ${ }^{20-21}$ For example, Auriol et al. ${ }^{22}$ reported that the comparative estrogenic activities of steroid estrogens are 2-3 orders of magnitude higher than other endocrine disrupters like phytoestrogens.

\subsection{Sunscreens}

Sunscreens are a group of products used to prevent or reduce the harm on human health from solar irradiation. The key components of sunscreen are UV-filters, which can absorb solar irradiation ranging from 280 to $320 \mathrm{~nm}$. UV-filters include a collection of organic (e.g., benzophenone and camphor derivatives) and inorganic substances (e.g., titanium dioxide and zinc oxide). Many studies have demonstrated that UV-filters could inhibit the growth of marine phytoplankton. ${ }^{23-25}$ However, relatively scarce occurrence data of UV-filters are available up to date. Table S1 shows the two most commonly detected UV-filters in coastal waters, which may be more vulnerable to sunscreen contamination due to growing coastal tourism and coastal-zone 
population. ${ }^{26}$

\subsection{Other PPCPs}

Two illicit drugs, caffeine, and cocaine are frequently detected in various water compartments. Caffeine is the most widely consumed psychoactive drug all over the world, and it was sometimes reported to be the predominant PPCP in several screening-level studies. ${ }^{27-28}$ Septic tanks and WWTPs effluents are the main source for caffeine, and its maximum concentration is recorded in groundwaters, reaching a high $\mu \mathrm{g} / \mathrm{L}$ level. Carbamazepine is a frequently detected antiepileptic

drug, with a maximum concentration of $99 \mu \mathrm{g} / \mathrm{L}$ reported in a groundwater. ${ }^{29}$ It can also be used as a marker to indicate human activity in the environments. DEET is an active component in insect repellents, and it has a relatively low ADI value among this subgroup of PPCPs. Triclosan (TCS) is a broad-spectrum antimicrobial and preservative agent with a considerable annual production. It was reported that more than 300 tons of TCS was disposed into wastewater every year in the U.S. ${ }^{30}$ TCS is widely detected especially in surface waters with concentrations reaching several $\mu \mathrm{g} / \mathrm{L}$. The ADI values of carbamazepine, DEET and TCS are lower than other ones except hormones, and the detection frequencies and occurrence concentrations of these PPCPs are also high. This indicates that special attention should be paid on them due to their potentially high-risk levels on human health.

\section{Pesticides}

Pesticides are used to control weeds, insects, and other pests in agricultural and urban areas, aiming to increase food production and reduce insect diseases. According to target pests, pesticides are classified into four subclasses, namely herbicide, insecticide, fungicide, and a mixed group including fumigants, nematicides, and other pesticides. These subclasses consist of a variety of compounds with different chemical makeups, including carbamates and organophosphates. ${ }^{31}$ Here we summarize related properties of herbicides and insecticides due to their relatively large usage. Numerous studies have reported the detection of herbicides and insecticides in aquatic environments, where they may impact aquatic organisms and human health when their 
concentrations reach toxic levels. ${ }^{32-34}$ In addition, the occurrence of pesticides has distinct seasonal patterns mainly due to timing of application and rainstorms, usually characterized by long periods of low concentrations while punctuated by much higher concentrations in sowing season. ${ }^{35}$ As shown in Table S2, the occurrence levels of pesticides basically lie between $\mathrm{ng} / \mathrm{L}$ to low $\mu \mathrm{g} / \mathrm{L}$, and the detection frequencies are relatively low compared to those of PPCPs. Notably, the maximum concentrations of most pesticides are greater than their PNEC values, which indicates that the recorded pesticides may cause damage to the aquatic ecosystems. Moreover, the occurrence levels of pesticides varied largely with water compartments. Surface waters are more vulnerable to be contaminated by pesticides than groundwaters, as signified by much higher detection frequencies and maximum concentrations of most pesticides in surface waters. In terms of the comparison between herbicides and insecticides, herbicides usually have higher detection frequencies than insecticides, probably due to their higher usage. Although many pesticides have maximum concentrations higher than their corresponding PNEC values, they seldom occurred at levels that are able to impact human health.

\subsection{Herbicides}

Herbicides are mainly used to kill undesired plants, but they have little impacts on the growth of wanted crops. They have considerable higher application amounts compared with other pesticides. Cyanazine, diuron, and isoproturon are among the three most frequently detected herbicides based on the results of Table S2, indicating their wide distribution in aquatic environments. By contrast, atrazine and metolachlor have relatively high maximum concentrations. Atrazine has a higher detection frequency than metolachlor in surface waters and groundwaters, possibly because of its stronger resistance to degradation. It was reported that the half-lifes of atrazine and metolachlor in soil were 146 and 26 days, respectively. ${ }^{31}$ The lowest PNEC and ADI values are recorded for bromacil $(6.8 \mathrm{ng} / \mathrm{L})$ and $\mathrm{MCPP}\left(1 \times 10^{-3} \mathrm{mg} / \mathrm{kg}\right.$-day $)$. The maximum concentration of bromacil in surface waters is recorded to be $390 \mathrm{ng} / \mathrm{L}$, which is indicative of its high-risk level on aquatic environments.

\subsection{Insecticides}


Insecticide are credited to be a great invention contributing to the increase of agricultural productivity in last century. Organochlorines, organophosphates, and pyrethroids are three commonly used kinds of insecticides, ${ }^{20}$ all of which are highly persistent in environments. For instance, the agricultural use of dichlorodiphenyltrichloroethane (DDT) has been banned for use since 1972, while it still can be detected, with a maximum concentration of $900 \mathrm{ng} / \mathrm{L}$. Compared with herbicides, most insecticides are more hydrophobic, leading to a higher partition of insecticides in riverbeds than in streams. The highest detection frequency and maximum concentration are recorded for carbaryl $(16.5 \%)$ and endosulfan $(1.8 \mu \mathrm{g} / \mathrm{L})$. Organochlorines and organophosphates are also well known by their high toxicity. According to Table S2, two insecticides, i.e., methoxychlor (orhanochlorines) and ethion (organophosphates), have relatively low PNEC values, which are at least one order of magnitude lower than those of other insecticides. The wide application of such toxic insecticides in last century had substantially adverse impacts on environments, and eventually led to prohibition of further usage of these insecticides. Pyrethroid insecticides have relatively low toxicity compared with organochlorine and organophosphate insecticides, and they have been increasingly used as their substitutes. ${ }^{36}$

\section{Industrial chemicals}

Industrial chemicals are defined here as any organic compounds manufactured or used, coupled with their byproducts, educts, and intermediates in industrial production processes. Rapid industrialization in recent decades has largely increased the production of industrial chemicals, and also resulted in deterioration of aquatic environments. ${ }^{37}$ Industrial chemicals include diverse compounds with substantially different chemical compositions. Industrial chemicals can be divided into seven subgroups, including phenols, polycyclic aromatic hydrocarbons (PAHs), triazoles, per- and poly-fluoroalkyl substances (PFAS), polybrominated diphenyl ethers (PBDEs), phthalate, and antioxidants. The occurrence and hazard parameters of industrial chemicals are summarized in Table S3. Typical occurrence concentrations of industrial chemicals lie in the range of $\mathrm{ng} / \mathrm{L}$ to $\mu \mathrm{g} / \mathrm{L}$. The highest concentration is recorded at $98 \mu \mathrm{g} / \mathrm{L}$ for bis(2-ethylhexyl) phthalate, 
and its detection frequency is also the highest. Several industrial chemicals like PFOS and PFOA have high PNEC values and relatively low ADI values, indicating their different toxic levels on environments and human health. BDE 99 has the lowest PNEC and ADI values, suggesting its high priority for research study when toxicity is considered only. But the maximum concentration of BDE 99 is only $11 \mathrm{ng} / \mathrm{L}$, which compromises the prioritization for BDE 99.

\subsection{Phenols}

Table S3 shows the occurrence and hazard information of four commonly detected phenols, including nonylphenol, bisphenol A (BPA), tert-octylphenol, and 4-nitrophenol. Alkylphenols such as nonylphenol and tert-octylphenol are often used as surfactant and household cleaners. ${ }^{38}$ They are endocrine disrupting compounds (EDCs) as well, although their effects are usually several orders of magnitude lower than hormones. ${ }^{39}$ BPA is a starting material for plastics and has been made into a collection of common consume products. BPA can bind into estrogen receptors, therefore it is an EDC as well. ${ }^{40}$ 4-Nitrophenol is a frequently used industrial feedstock in synthesis processes, and it can also be used as a $\mathrm{pH}$ indicator. ${ }^{41}$

\subsection{PAHs}

PAHs are characterized by their uncharged, non-polar multiple aromatic rings, which are mainly produced from incomplete incineration of organic matter in both industrial and natural processes. PAHs are typically enriched in the river sediment, ${ }^{42}$ and several PAHs including pyrene, benz(a)anthracene and naphthalene are also detected at high concentrations in aquatic environments. These PAHs have relatively low molecular weights, because the aqueous solubility of a PAH usually decreases significantly as its molecular weight increases. ${ }^{43}$ By contrast, PAHs with high molecular weights were reported to have high risks on aquatic organisms especially invertebrates. ${ }^{44}$ These PAHs can be used as material feedstock, and pyrene is also a probe to detect solvents due to its sensitive fluorescence spectrum. The maximum concentration of pyrene reaches high ng/L level, which is higher than its PNEC value. However, ADI values of PAHs are not well established.

\subsection{Triazoles}


Benzotriazoles and tolytriazoles are used as corrosion inhibitors in a variety of products, such as dishwasher detergents, engine coolants, and aircraft deicers. ${ }^{45}$ They have high detection frequencies and maximum concentrations, suggesting their wide distribution in aquatic environments. However, their PNEC and ADI values are also relatively high, which may lower their risk levels on environments and human health.

\subsection{PFASs}

PFASs are featured by their unique property of repelling both lipids and water, which enables them to be used as liquid repellents and grease-proof coatings. PFOS and PFOA are among the PFASs commonly detected at relatively high levels in the aquatic ecosystems, ${ }^{46}$ and PFOS was also reported to have a high bioconcentration potential in fish ${ }^{47}$ As shown in Table S3, these two compounds have high detection frequencies especially in surface waters. The maximum concentration of PFOS reaches a low $\mu \mathrm{g} / \mathrm{L}$ level in surface waters, which is even comparable to its PNEC value.

\subsection{PBDEs}

PBDEs are commonly used flame retardants that have wide applications on our daily products like furniture, building materials, and computers. The occurrence levels of PBDEs in aquatic environments are very low, nevertheless, concerns still arise because of their robust persistence in aquatic environments and high bioaccumulation potential. ${ }^{48}$ In addition, two PBDEs, BDE 47 and BDE 99, have the lowest PNEC and ADI values among industrial chemicals recorded in Table S3. The major concerns induced by PBDEs include hormonal disruption, cancer, and developmental neurotoxicity. ${ }^{49}$

\subsection{Phthalates}

Phthalates are mainly used to modify many properties of plastic, like flexibility, transparency, durability, and longevity. ${ }^{50}$ The most widely used phthalates include diethyl phthalate, dioctyl phthalate, bis(2-ethylhexyl) phthalate, and di-n-butyl phthalate. Bis(2-ethylhexyl) phthalate is among the most frequently detected phthalates in aquatic environments, and its maximum concentration reaches the $\mu \mathrm{g} / \mathrm{L}$ level. By contrast, other phthalates have relatively low detection 
frequencies and occurrence levels.

\subsection{Antioxidants}

Substances in this group mainly function as preservatives in a range of foods, cosmetics, and fuels. Butylated hydroxyanisole and butylated hydroxytoluene are the two commonly used antioxidants. Butylated hydroxyanisole was reported to be toxic on bacteria, algae, and other aquatic organisms, and it was also detectable in drinking waters. ${ }^{51}$ However, the PNEC and ADI values of these two antioxidants are not well-established. 
Table S1. The concentrations, PNEC and ADI values of the commonly detected PPCPs in various water bodies.

\begin{tabular}{|c|c|c|c|c|c|c|c|c|}
\hline Subgroup & Compound & Water type & $\begin{array}{c}\text { Detection } \\
\text { frequency } \\
(\%)\end{array}$ & $\begin{array}{c}\text { Med or } \\
\text { mean conc } \\
(\mathrm{ng} / \mathrm{L})\end{array}$ & $\begin{array}{c}\text { Max } \\
\text { conc } \\
\text { (ng/L) } \\
\end{array}$ & $\begin{array}{l}\text { PNEC } \\
(\mathrm{ng} / \mathrm{L})\end{array}$ & $\begin{array}{c}\text { ADI } \\
\text { (mg/kg- } \\
\text { day) }\end{array}$ & $\begin{array}{l}\text { Reference } \\
\text { code * }\end{array}$ \\
\hline \multirow[t]{16}{*}{ Antibiotics } & Erythromycin & Surface water & 57 & & 1540 & 20 & $4.0 \mathrm{E}-02$ & $\mathrm{C}, \mathrm{B}, \mathrm{Sc}$ \\
\hline & Amoxicillin & Surface water & & 240 & 2340 & 37 & & $\mathrm{P}, \mathrm{V}$ \\
\hline & Trimethoprim & Coastal water & 80 & 5.15 & 18 & 6000 & $9.4 \mathrm{E}-03$ & F, M, B \\
\hline & \multirow{2}{*}{ Sulfamethazine } & Surface water & 80 & & 110 & \multirow{2}{*}{3000} & \multirow{2}{*}{$1.6 \mathrm{E}-03$} & $\mathrm{~B}, \mathrm{Ta}, \mathrm{Le}$ \\
\hline & & Coastal water & 97 & 0.92 & 1.5 & & & $\mathrm{~F}$ \\
\hline & \multirow{2}{*}{ Lincomycin } & Coastal water & 81 & 4.28 & 7.6 & \multirow{2}{*}{82000} & \multirow{2}{*}{$2.5 \mathrm{E}-02$} & $\mathrm{~F}, \mathrm{~A}, \mathrm{Sc}$ \\
\hline & & Groundwater & & 188 & 320 & & & $\mathrm{La}$ \\
\hline & Ofloxacin & Surface water & & 645 & 2028 & 16 & & $\mathrm{D}, \mathrm{A}$ \\
\hline & \multirow{3}{*}{ Sulfamethoxazole } & Surface water & 100 & 86.2 & 114.2 & \multirow{3}{*}{27} & \multirow{3}{*}{$1.3 \mathrm{E}-01$} & So, B, La \\
\hline & & Coastal water & 100 & 26 & 220 & & & F \\
\hline & & Groundwater & 24.2 & 252 & 1110 & & & $\mathrm{La}$ \\
\hline & Sulfamerazine & Coastal water & 97 & 0.92 & 1.5 & 1800000 & & $\mathrm{~F}, \mathrm{Le}$ \\
\hline & Roxithromycin & Surface water & 91 & & 3700 & 100 & $4.0 \mathrm{E}-04$ & $\mathrm{~B}, \mathrm{Le}$ \\
\hline & Ciprofloxacin & Surface water & 100 & 155 & 299.8 & 2000 & $1.6 \mathrm{E}-03$ & So, B, Sc \\
\hline & Clarithromycin & Surface water & 30 & 1.5 & 170 & 70 & $2.0 \mathrm{E}-04$ & $\mathrm{~A}, \mathrm{C}, \mathrm{Le}$ \\
\hline & Tetracycline & Surface water & 1.2 & 140 & 1300 & 90 & $3.0 \mathrm{E}-02$ & $\mathrm{~V}, \mathrm{~A}, \mathrm{Sc}$ \\
\hline \multirow{3}{*}{$\begin{array}{l}\text { Lipid } \\
\text { regulators }\end{array}$} & Clofibric acid & Groundwater & 81.4 & 1113 & 7300 & 24600 & $1.0 \mathrm{E}-02$ & $\mathrm{La}, \mathrm{Mu}$ \\
\hline & \multirow{2}{*}{ Gemfibrozil } & Coastal water & 98 & 7.03 & 49 & \multirow{2}{*}{150} & \multirow{2}{*}{$1.3 \mathrm{E}-03$} & $\mathrm{~F}, \mathrm{Mu}$ \\
\hline & & Surface water & 35 & 29 & 970 & & & Lo \\
\hline \multirow{13}{*}{$\begin{array}{l}\text { Analgesics } \\
\text { and NSAIDs }\end{array}$} & Acetaminophen & Surface water & 23.8 & 110 & 10000 & 9200 & 3.4E-01 & $\mathrm{Mu}, \mathrm{Y}$ \\
\hline & Phenazone & Groundwater & & 1503 & 3950 & 1100 & & $\mathrm{La}, \mathrm{V}$ \\
\hline & Salicylic acid & Groundwater & & 418 & 1225 & 10000 & $2.6 \mathrm{E}-03$ & $\mathrm{La}, \mathrm{Le}$ \\
\hline & Codeine & Surface water & & 1700 & 35000 & 16000 & $2.0 \mathrm{E}-03$ & $\mathrm{~A}, \mathrm{Sc}$ \\
\hline & \multirow{2}{*}{ Diclofenac } & Surface water & 83 & 432 & 1043 & \multirow{2}{*}{9700} & \multirow{2}{*}{$6.7 \mathrm{E}-02$} & St, So, A, B \\
\hline & & Groundwater & & 121 & 590 & & & $\mathrm{La}$ \\
\hline & \multirow{3}{*}{ Ibuprofen } & Surface water & 62 & 19.5 & 67 & \multirow{3}{*}{1000} & \multirow{3}{*}{$1.1 \mathrm{E}-01$} & $\mathrm{St}, \mathrm{Lo}, \mathrm{Sc}$ \\
\hline & & Coastal water & 31 & 7.88 & 84 & & & $\mathrm{~F}$ \\
\hline & & Groundwater & 6.7 & 1491 & 12000 & & & $\mathrm{La}, \mathrm{B}$ \\
\hline & & Groundwater & & 611 & 2886 & & 5.7E-01 & $\mathrm{La}, \mathrm{Vo}$ \\
\hline & Ketoprofen & Surface water & 25 & 60 & 395 & 12130 & & St \\
\hline & & Surface water & 62.5 & 106 & 322 & & & St, B, A \\
\hline & Naproxen & Coastal water & 100 & 6.32 & 30 & 2620 & & $\mathrm{~F}$ \\
\hline Hormones & & Surface water & 17 & 0.47 & 5.8 & & & $\mathrm{M}, \mathrm{Ya}, \mathrm{Mu}$ \\
\hline & Estrone (E1) & & & & & 3.6 & $1.3 \mathrm{E}-05$ & \\
\hline & & Groundwater & & 9 & 45 & & & $\mathrm{La}$ \\
\hline & & Surface water & 10.6 & 9 & 93 & & & $\mathrm{M}, \mathrm{Mu}$ \\
\hline & Estradiol (E2) & Groundwater & & 31 & 120 & 0.4 & $5.0 \mathrm{E}-06$ & $\mathrm{La}$ \\
\hline & Estriol (E3) & Surface water & 21.4 & 19 & 51 & 60 & & $\mathrm{M}, \mathrm{Mu}$ \\
\hline & $\begin{array}{c}\text { Ethinyloestradiol } \\
\text { (EE2) }\end{array}$ & Surface water & 15.7 & 73 & 831 & 0.035 & $1.0 \mathrm{E}-07$ & $\mathrm{Ca}, \mathrm{Mu}$ \\
\hline Sunscreen & benzophehone 3 & Coastal water & & 210.2 & 577.5 & 360 & & $\mathrm{R}$, To \\
\hline & benzophehone 4 & Surface water & & 168 & 373 & 4896 & & $\mathrm{R}$ \\
\hline Other PPCPs & & Surface water & 100 & 30.82 & 36.75 & & & So, Y, Le \\
\hline & Caffeine & Coastal water & 44 & 31 & 100 & 2460 & $1.5 \mathrm{E}-01$ & $\mathrm{~F}$ \\
\hline & & Groundwater & 82.9 & 9774 & 110000 & & & $\mathrm{La}$ \\
\hline & Cocaine & Surface water & 75 & 2.8 & 34.2 & & & $\mathrm{M}, \mathrm{Ma}$ \\
\hline & & Coastal water & 30 & 2.23 & 10 & & & $\mathrm{~F}, \mathrm{Mu}, \mathrm{Le}$ \\
\hline & Carbamazepine & Groundwater & 42.1 & 5312 & 99194 & 13800 & $3.0 \mathrm{E}-04$ & $\mathrm{La}$ \\
\hline
\end{tabular}




\section{Table S1 (Continued)}

\begin{tabular}{|c|c|c|c|c|c|c|c|c|}
\hline Subgroup & Compound & Water type & $\begin{array}{c}\text { Detection } \\
\text { frequency } \\
(\%)\end{array}$ & $\begin{array}{c}\text { Med or } \\
\text { mean conc } \\
(\mathrm{ng} / \mathrm{L})\end{array}$ & $\begin{array}{c}\text { Max } \\
\text { conc } \\
(\mathrm{ng} / \mathrm{L})\end{array}$ & $\begin{array}{l}\text { PNEC } \\
\text { (ng/L) }\end{array}$ & $\begin{array}{c}\text { ADI } \\
\text { (mg/kg- } \\
\text { day) }\end{array}$ & $\begin{array}{c}\text { Reference } \\
\text { code * }\end{array}$ \\
\hline \multirow[t]{7}{*}{ Other PPCPs } & Carbamazepine & Surface water & 95 & 248 & 11561 & & & Lo \\
\hline & $\begin{array}{l}\text { N,N-Diethyl- } \\
\text { meta-toluamide } \\
(\mathrm{DEET})\end{array}$ & Groundwater & 10 & 2251 & 6500 & & $8.2 \mathrm{E}-05$ & $\mathrm{La}, \mathrm{Mu}$ \\
\hline & Fluoxetine & Surface water & 100 & 11.13 & 37.13 & 24 & $2.9 \mathrm{E}-03$ & $\mathrm{Ya}, \mathrm{A}, \mathrm{Sc}$ \\
\hline & Propranolol & Surface water & 90 & 2.72 & 10.83 & 244 & & $\mathrm{C}$ \\
\hline & Sertraline & Surface water & 100 & 9.16 & 53.17 & 48 & & Ya \\
\hline & \multirow[t]{2}{*}{ Triclosan } & Surface water & 95.8 & 26.75 & 98 & \multirow[t]{2}{*}{1200} & \multirow[t]{2}{*}{$1.9 \mathrm{E}-04$} & St, So, Mu \\
\hline & & Groundwater & 50 & 509 & 2110 & & & $\mathrm{La}$ \\
\hline
\end{tabular}

* full references are listed below table S3 
Table S2. The concentrations, PNEC and ADI values of the commonly detected pesticides in various water bodies.

\begin{tabular}{|c|c|c|c|c|c|c|c|c|}
\hline Subgroup & Compound & Water type & $\begin{array}{c}\text { Detection } \\
\text { frequency } \\
(\%)\end{array}$ & $\begin{array}{c}\text { Med or } \\
\text { mean conc } \\
(\mathrm{ng} / \mathrm{L})\end{array}$ & $\begin{array}{c}\text { Max } \\
\text { conc } \\
(n g / L)\end{array}$ & $\begin{array}{c}\text { PNEC } \\
(\mathrm{ng} / \mathrm{L})\end{array}$ & $\begin{array}{l}\text { ADI (mg } \\
\text { / kg-day) }\end{array}$ & $\begin{array}{l}\text { Reference } \\
\text { Code * }\end{array}$ \\
\hline \multirow[t]{19}{*}{ Herbicide } & 2,4-D & Surface water & 52 & 3 & 1200 & & $1.0 \mathrm{E}-02$ & U, Vo \\
\hline & & Groundwater & 2.1 & & 30 & & & M \\
\hline & Acetochlor & Surface water & 48 & 100 & 200 & & $2.0 \mathrm{E}-02$ & $\mathrm{U}, \mathrm{V}_{\mathrm{O}}$ \\
\hline & Alachlor & Surface water & 7.5 & 50 & 630 & 300 & $1.0 \mathrm{E}-02$ & U \\
\hline & Atrazine & Surface water & 68 & 2000 & 2100 & 600 & $3.5 \mathrm{E}-02$ & $\mathrm{U}$ \\
\hline & & Groundwater & 34.2 & & 4 & & & M \\
\hline & Bentazone & Surface water & 69 & 4 & 250 & 100 & $3.0 \mathrm{E}-02$ & $\mathrm{U}$ \\
\hline & & Groundwater & 5.6 & & 30 & & & $\mathrm{M}$ \\
\hline & Bromacil & Surface water & 21.7 & & 390 & 6.8 & & $\mathrm{Mu}, \mathrm{Vo}$ \\
\hline & Cyanazine & Surface water & 70 & 100 & 300 & 12 & & $\mathrm{U}, \mathrm{W}$ \\
\hline & Diuron & Surface water & 70 & 10 & 860 & 200 & $2.0 \mathrm{E}-03$ & $\mathrm{U}$ \\
\hline & & Groundwater & 7.5 & & 20 & & & M \\
\hline & Isoproturon & Surface water & 70 & 4 & 2000 & 300 & $3.0 \mathrm{E}-03$ & $\mathrm{U}$ \\
\hline & МСРP & Surface water & 43 & 15 & 190 & & $1.0 \mathrm{E}-03$ & $\mathrm{U}$ \\
\hline & Metolachlor & Surface water & 39.2 & 1000 & 11000 & 70 & $1.5 \mathrm{E}-01$ & $\mathrm{U}$ \\
\hline & & Groundwater & 8.9 & & 10 & & & M \\
\hline & Prometon & Groundwater & 25.7 & & 1000 & 100 & $1.5 \mathrm{E}-02$ & $\mathrm{U}$ \\
\hline & Simazine & Surface water & 26 & 10 & 170 & 1000 & $5.0 \mathrm{E}-03$ & $\mathrm{U}$ \\
\hline & & Groundwater & 20.2 & & 6 & & & M \\
\hline \multirow[t]{15}{*}{ Insecticide } & Aldrin & Surface water & & & & 10 & $5.0 \mathrm{E}-05$ & $\mathrm{U}$ \\
\hline & Biphenthrin & Surface water & & & & & $1.5 \mathrm{E}-02$ & $\mathrm{U}$ \\
\hline & Carbaryl & Groundwater & 16.5 & 40 & 100 & 10 & $1.0 \mathrm{E}-01$ & $\mathrm{U}$ \\
\hline & Chlorpyrifos & Groundwater & 15.3 & 60 & 310 & 30 & $3.0 \mathrm{E}-03$ & $\mathrm{U}$ \\
\hline & Cypermethrin & Surface water & & & & & $1.0 \mathrm{E}-02$ & $\mathrm{U}$ \\
\hline & DDT & Surface water & 10 & 180 & 900 & 10 & $5.0 \mathrm{E}-04$ & $\mathrm{U}$ \\
\hline & Diazinon & Groundwater & 13 & 70 & 1100 & 17 & $9.0 \mathrm{E}-05$ & $\mathrm{U}, \mathrm{Te}, \mathrm{Vo}$ \\
\hline & Dieldrin & Groundwater & 4.7 & 180 & 210 & 10 & $5.0 \mathrm{E}-05$ & $\mathrm{U}$ \\
\hline & Endosulfan & Surface water & 8 & & 1800 & 5 & $6.0 \mathrm{E}-03$ & U \\
\hline & Esfenvalerate & Surface water & & & 140 & & $2.0 \mathrm{E}-02$ & $\mathrm{U}$ \\
\hline & Ethion & Surface water & & & & 0.56 & $5.0 \mathrm{E}-05$ & U \\
\hline & Heptachlor & Surface water & 3 & & 240 & 33 & $5.0 \mathrm{E}-04$ & $\mathrm{U}, \mathrm{Sn}$ \\
\hline & Lindane & Surface water & 3 & 20 & 200 & 20 & $5.6 \mathrm{E}-04$ & $\mathrm{U}, \mathrm{Sn}$ \\
\hline & Malathion & Surface water & 4 & & & 6 & $2.0 \mathrm{E}-02$ & U \\
\hline & Methoxychlor & Surface water & 1 & 100 & 1700 & 0.5 & $2.0 \mathrm{E}-04$ & $\mathrm{U}$ \\
\hline
\end{tabular}

* full references are listed below table S3 
Table S3. The concentrations, PNEC and ADI values of the commonly detected industrial chemicals in various water bodies.

\begin{tabular}{|c|c|c|c|c|c|c|c|c|c|}
\hline Subgroup & Compound & Common use & Water type & $\begin{array}{c}\text { Detection } \\
\text { frequency } \\
(\%) \\
\end{array}$ & $\begin{array}{c}\text { Med or } \\
\text { mean conc } \\
(\mathrm{ng} / \mathrm{L})\end{array}$ & $\begin{array}{c}\text { Max } \\
\text { conc } \\
(\mathrm{ng} / \mathrm{L})\end{array}$ & $\begin{array}{c}\text { PNEC } \\
(\mathrm{ng} / \mathrm{L})\end{array}$ & $\begin{array}{l}\text { ADI (mg } \\
\text { / kg-day) }\end{array}$ & $\begin{array}{c}\text { Reference } \\
\text { code }\end{array}$ \\
\hline \multirow[t]{5}{*}{ Phenol } & \multirow{2}{*}{ nonylphenol } & \multirow{2}{*}{ surfactant } & surface water & 50.6 & 800 & 40000 & \multirow{2}{*}{210} & \multirow{2}{*}{$5.00 \mathrm{E}-02$} & $\mathrm{C}, \mathrm{K}$ \\
\hline & & & drinking water & 21 & 110 & 130 & & & $\mathrm{Sn}$ \\
\hline & bisphenol A & plasticizer & surface water & 41.2 & 140 & 12000 & 11000 & $5.00 \mathrm{E}-02$ & $\mathrm{C}, \mathrm{K}, \mathrm{U}$ \\
\hline & tert-octylphenol & $\begin{array}{l}\text { surfactant, household } \\
\text { cleaners }\end{array}$ & surface water & 8 & 13 & 560 & 48 & $1.50 \mathrm{E}-01$ & $\mathrm{R}, \mathrm{K}$ \\
\hline & 4-Nitrophenol & material feedstock & surface water & 97 & 16 & 3471 & 3800 & & Ch, Lo \\
\hline \multirow[t]{4}{*}{ PAHs } & pyrene & material feedstock & surface water & 28.2 & 50 & 840 & 40 & & $\mathrm{G}, \mathrm{K}$ \\
\hline & benz(a)anthracen & & surface water & 11.8 & 10 & 530 & & & $\mathrm{~K}$ \\
\hline & $\mathrm{e}$ & material feedstock & groundwater & 92 & 1.4 & 1.8 & 300 & & $\mathrm{Li}$ \\
\hline & naphthalene & material feedstock & surface water & 16.5 & 20 & 80 & 1200 & & $\mathrm{G}, \mathrm{K}$ \\
\hline \multirow[t]{3}{*}{ Triazoles } & & & surface water & 94 & 230 & 8000 & \multirow{2}{*}{20000} & \multirow[b]{2}{*}{$3.00 \mathrm{E}-01$} & $\mathrm{Mz}, \mathrm{K}$ \\
\hline & benzotriazole & corrosion inhibitors & groundwater & & 37 & 53 & & & $\mathrm{Li}$ \\
\hline & tolyltriazole & corrosion inhibitors & surface water & 81 & 140 & 19396 & 3851 & $2.50 \mathrm{E}-01$ & $\mathrm{Ku}, \mathrm{Lo}$ \\
\hline \multirow[t]{4}{*}{ PFAS } & perfluorooctanoa & surfactant, water & surface water & 97 & 3 & 174 & \multirow{2}{*}{113022} & \multirow{2}{*}{$1.50 \mathrm{E}-03$} & R, Lo \\
\hline & te $(\mathrm{PFOA})$ & proofing & groundwater & 85 & 0.18 & 7.1 & & & $\mathrm{Li}$ \\
\hline & perfluorooctansu & \multirow{2}{*}{ water proofing } & surface water & 94 & 6 & 1371 & \multirow{2}{*}{1080} & \multirow{2}{*}{$1.50 \mathrm{E}-04$} & R, Lo \\
\hline & lfonate (PFOS) & & groundwater & 9 & & 0.21 & & & $\mathrm{Li}$ \\
\hline \multirow[t]{3}{*}{ PBDEs } & $\begin{array}{c}2,20,4,40- \\
\text { Tetrabromodiph } \\
\text { enyl ether (BDE- } \\
47)\end{array}$ & flame retardant & marine water & & & 5 & 0.6 & $4.00 \mathrm{E}-04$ & G, Vo, U \\
\hline & $2,20,4,40,5-$ & & & & & & & & \\
\hline & $\begin{array}{c}\text { Pentabromodiph } \\
\text { enyl ether (BDE- } \\
99)\end{array}$ & flame retardant & marine water & & & 11 & 0.5 & $1.00 \mathrm{E}-04$ & $\mathrm{G}, \mathrm{Vo}, \mathrm{U}$ \\
\hline \multirow[t]{5}{*}{ Phthalate } & \multirow{2}{*}{ diethyl phthalate } & \multirow{2}{*}{ plasticizer } & surface water & 11.1 & 200 & 420 & \multirow{5}{*}{1300} & \multirow{2}{*}{$8.00 \mathrm{E}-01$} & K \\
\hline & & & groundwater & 8 & 1040 & 2017 & & & $\mathrm{Li}$ \\
\hline & dioctyl phthalate & plasticizer & drinking water & 26 & 110 & 170 & & & Sn \\
\hline & bis(2-ethylhexyl) & & surface water & 99 & 2200 & 98000 & & \multirow{2}{*}{$2.00 \mathrm{E}-02$} & $\mathrm{Vo}, \mathrm{Fr}$ \\
\hline & phthalate & plasticizer & groundwater & 8 & 644 & 1144 & & & $\mathrm{Li}$ \\
\hline Phthalate & $\begin{array}{l}\text { Di-n-butyl } \\
\text { phthalate }\end{array}$ & plasticizer & surface water & 61.9 & 80 & 4920 & & $8.80 \mathrm{E}+00$ & $\mathrm{C}$ \\
\hline \multirow[t]{2}{*}{$\begin{array}{l}\text { antioxidan } \\
\text { ts }\end{array}$} & $\begin{array}{c}\text { butylated } \\
\text { hydroxyanisole }\end{array}$ & food additive & drinking water & 6 & 50 & 50 & & & $\mathrm{Sn}$ \\
\hline & $\begin{array}{c}\text { butylated } \\
\text { hydroxy toluene }\end{array}$ & food additive & surface water & 2.4 & 100 & 100 & & $3.00 \mathrm{E}-01$ & $\mathrm{~K}$ \\
\hline
\end{tabular}

C (Chau et al., 2018), B (Bu et al., 2013), Ca (Carlsson et al., 2006), Sc (Schwab et al., 2005), P (Perazzolo et al., 2010), V (Verlicchi et al., 2012), F (Ferguson et al., 2013), M (Munz et al., 2017), Ta (Tamtam et al., 2008), Le (Leung et al., 2013), A (Al Aukidy et al., 2014), La (Lapworth et al., 2012), D (Díaz-Garduño et al., 2017), So (Sodré et al., 2018), Mu (Murray et al., 2010), Lo (Loos et al., 2009), Y (Yang et al., 2017a), St (Stasinakis et al., 2012), Ya (Yan et al., 2018), R (Riva et al., 2019), To (Tovar-Sánchez et al., 2013), Ma (Ma et al., 2016), U (USEPA, 2010), W (WHO, 2002), Te (Teuschler et al., 1999), K (Kolpin et al., 2002), Sn (Snyder and Engineering, 2008), Ch (Cho et al., 2014), G (Ghekiere et al., 2013), Li (Li et al., 2016), Mz (Munz et al., 2018), Ku (Kuzmanović et al., 2015), Vo (von der Ohe et al., 2011), Fr (Fromme et al., 2002). 


\section{References}

(1) Ma, X.Y.; Li, Q.; Wang, X.C.; Wang, Y.; Wang, D.; Ngo, H.H. Micropollutants removal and health risk reduction in a water reclamation and ecological reuse system. Water Research. 2018, 138, 272-281.

(2) Petrie, B.; Barden, R.; Kasprzyk-Hordern, B. A review on emerging contaminants in wastewaters and the environment: current knowledge, understudied areas and recommendations for future monitoring. Water Research. 2015, 72, 3-27.

(3) Rivera-Utrilla, J.; Sánchez-Polo, M.; Ferro-García, M.Á.; Prados-Joya, G.; Ocampo-Pérez, R. Pharmaceuticals as emerging contaminants and their removal from water. A review. Chemosphere. 2013, 93(7), 1268-1287.

(4) Chopra, I.; Roberts, M. Tetracycline antibiotics: mode of action, applications, molecular biology, and epidemiology of bacterial resistance. Microbiology and Molecular Biology Reviews. 2001, 65(2), 232-260.

(5) Jones, O.; Voulvoulis, N.; Lester, J. Human pharmaceuticals in the aquatic environment a review. Environmental Technology. 2001, 22(12), 1383-1394.

(6) Bu, Q.; Wang, B.; Huang, J.; Deng, S.; Yu, G.J. Pharmaceuticals and personal care products in the aquatic environment in China: a review. Journal of Hazardous Materials. 2013, 262, 189-211.

(7) Daughton, C.G.; Ternes, T.A. Pharmaceuticals and personal care products in the environment: agents of subtle change? Environmental Health Perspectives. 1999, 107, 907-938.

(8) Tamtam, F.; Mercier, F.; Le Bot, B.; Eurin, J.; Dinh, Q.T.; Clément, M.; Chevreuil, M. Occurrence and fate of antibiotics in the Seine River in various hydrological conditions. Science of Total Environment. 2008, 393(1), 84-95.

(9) Isidori, M.; Lavorgna, M.; Nardelli, A.; Pascarella, L.; Parrella, A. Toxic and genotoxic evaluation of six antibiotics on non-target organisms. Science of Total Environment. 2005, 346(1-3), 87-98.

(10) Neumann, N.J.; Blotz, A.; Wasinska-Kempka, G.; Rosenbruch, M.; Lehmann, P.; Ahr, H.J.; Vohr, H. $\mathrm{W}$. Evaluation of phototoxic and photoallergic potentials of 13 compounds by different in vitro and in vivo methods. Journal of Photochemistry and Photobiology B: Biology. 2005, 79(1), 25-34.

(11) Sendzik, J.; Shakibaei, M.; Schäfer-Korting, M.; Stahlmann, R. Fluoroquinolones cause changes in extracellular matrix, signalling proteins, metalloproteinases and caspase-3 in cultured human tendon cells. Toxicology. 2005, 212(1), 24-36.

(12) Stan, H.; Heberer, T. Pharmaceuticals in the aquatic environment. Analusis. 1997, 7(25), M20-M23.

(13) Heberer, T. Occurrence, fate, and removal of pharmaceutical residues in the aquatic environment: a review of recent research data. Toxicology Letters. 2002, 131(1-2), 5-17.

(14) Kolpin, D.W.; Furlong, E.T.; Meyer, M.T.; Thurman, E.M.; Zaugg, S.D.; Barber, L.B.; Buxton, H.T. Pharmaceuticals, hormones, and other organic wastewater contaminants in US streams, 1999- 2000: A national reconnaissance. Environmental Science \& Technology. 2002, 36(6), 1202-1211.

(15) Ternes, T.A.; Kreckel, P.; Mueller, J. Behaviour and occurrence of estrogens in municipal sewage treatment plants-II. Aerobic batch experiments with activated sludge. Science of the Total Environment. 1999, 225(1-2), 91-99.

(16) Ternes, T. Pharmaceuticals and metabolites as contaminants of the aquatic environment: An overview. Abstracts of Papers of the American Chemical Society. 2000, 219, 622-623 
(17) Liu, Z.-h.; Ogejo, J.A.; Pruden, A.; Knowlton, K.F. Occurrence, fate and removal of synthetic oral contraceptives (SOCs) in the natural environment: A review. Science of Total Environment. 2011, 409(24), 5149-5161.

(18) Yang, Y.; Li, J.; Lu, K.; Shi, H.; Gao, S. Transformation of $17 \alpha$-ethinylestradiol by simultaneous photo-enzymatic process in Humic water. Chemosphere. 2017, 178, 432-438.

(19) Adeel, M.; Song, X.; Wang, Y.; Francis, D.; Yang, Y.J. Environmental impact of estrogens on human, animal and plant life: a critical review. Environment International. 2017, 99, 107-119.

(20) Murray, K.E.; Thomas, S.M.; Bodour, A.A. Prioritizing research for trace pollutants and emerging contaminants in the freshwater environment. Environmental Pollution. 2010, 158(12), 3462-3471.

(21) Yang, Y.; Li, J.; Shi, H.; Zhai, L.; Wang, X.; Gao, S.J. Influence of natural organic matter on horseradish peroxidase-mediated removal of $17 \alpha$-ethinylestradiol: Role of molecular weight. Journal of Hazardous Materials. 2018, 356, 9-16.

(22) Auriol, M.; Filali-Meknassi, Y.; Adams, C.D.; Tyagi, R.D. Natural and synthetic hormone removal using the horseradish peroxidase enzyme: temperature and pH effects. Water Research. 2006, 40(15), 28472856.

(23) Gao, L.; Yuan, T.; Zhou, C.; Cheng, P.; Bai, Q.; Ao, J.; Wang, W.; Zhang, H. Effects of four commonly used UV filters on the growth, cell viability and oxidative stress responses of the Tetrahymena thermophila. Chemosphere. 2013, 93(10), 2507-2513.

(24) Minetto, D.; Ghirardini, A.V.; Libralato, G. Saltwater ecotoxicology of Ag, Au, CuO, TiO2, ZnO and C60 engineered nanoparticles: an overview. Environment International. 2016, 92, 189-201.

(25) Paredes, E.; Pérez, S.; Rodil, R.; Quintana, J.B.; Beiras, R. Ecotoxicological evaluation of four UV filters using marine organisms from different trophic levels Isochrysis galbana, Mytilus galloprovincialis, Paracentrotus lividus, and Siriella armata. Chemosphere. 2014, 104, 44-50.

(26) Sánchez-Quiles, D.; Tovar-Sánchez, A. Are sunscreens a new environmental risk associated with coastal tourism? Environment International. 2015, 83, 158-170.

(27) Ma, R.; Wang, B.; Lu, S.; Zhang, Y.; Yin, L.; Huang, J.; Deng, S.; Wang, Y.; Yu, G. Characterization of pharmaceutically active compounds in Dongting Lake, China: occurrence, chiral profiling and environmental risk. Science of Total Environment. 2016, 557, 268-275.

(28) Wu, C.; Huang, X.; Witter, J.D.; Spongberg, A.L.; Wang, K.; Wang, D.; Liu, J. Occurrence of pharmaceuticals and personal care products and associated environmental risks in the central and lower Yangtze river, China. Ecotoxicology and Environmental Safety. 2014, 106, 19-26.

(29) Lapworth, D.; Baran, N.; Stuart, M.; Ward, R. Emerging organic contaminants in groundwater: a review of sources, fate and occurrence. Environmental Pollution. 2012, 163, 287-303.

(30) Halden, R.U.; Paull, D.H. Co-occurrence of triclocarban and triclosan in US water resources. Environmental Science \& Technology. 2005, 39(6), 1420-1426.

(31) Gilliom, R.J.; Barbash, J.E.; Crawford, C.G.; Hamilton, P.A.; Martin, J.D.; Nakagaki, N.; Nowell, L.H.; Scott, J.C.; Stackelberg, P.E.; Thelin, G.P. Pesticides in the nation's streams and ground water, 19922001, US Geological Survey. 2006.

(32) Konstantinou, I.K.; Hela, D.G.; Albanis, T.A. The status of pesticide pollution in surface waters (rivers 
and lakes) of Greece. Part I. Review on occurrence and levels. Environmental Pollution. 2006, 141(3), 555570.

(33) Solomon, K.R.; Baker, D.B.; Richards, R.P.; Dixon, K.R.; Klaine, S.J.; La Point, T.W.; Kendall, R.J.; Weisskopf, C.P.; Giddings, J.M.; Giesy, J.P. Ecological risk assessment of atrazine in North American surface waters. Environmental Toxicology and Chemistry. 1996, 15(1), 31-76.

(34) Weber, J.; Halsall, C.J.; Muir, D.; Teixeira, C.; Small, J.; Solomon, K.; Hermanson, M.; Hung, H.; Bidleman, T. Endosulfan, a global pesticide: A review of its fate in the environment and occurrence in the Arctic. Science of the Total Environment. 2010, 408(15), 2966-2984.

(35) von der Ohe, P.C.; Dulio, V.; Slobodnik, J.; De Deckere, E.; Kühne, R.; Ebert, R.-U.; Ginebreda, A.; De Cooman, W.; Schüürmann, G.; Brack, W. A new risk assessment approach for the prioritization of 500 classical and emerging organic microcontaminants as potential river basin specific pollutants under the European Water Framework Directive. Science of the Total Environment. 2011, 409(11), 2064-2077.

(36) Brady, J.; Wallender, W.; Werner, I.; Fard, B.M.; Zalom, F.; Oliver, M.; Wilson, B.; Mata, M.; Henderson, J.; Deanovic, L. Pesticide runoff from orchard floors in Davis, California, USA: a comparative analysis of diazinon and esfenvalerate. Agriculture, Ecosystem \& Environment. 2006, 115(1-4), 56-68.

(37) Dsikowitzky, L.; Schwarzbauer, J. Industrial organic contaminants: identification, toxicity and fate in the environment. Environmental Chemistry Letters. 2014, 12(3), 371-386.

(38) Tan, B.L.; Hawker, D.W.; Müller, J.F.; Leusch, F.D.; Tremblay, L.A.; Chapman, H.F.JModelling of the fate of selected endocrine disruptors in a municipal wastewater treatment plant in South East Queensland, Australia. Chemosphere. 2007, 69(4), 644-654.

(39) Gasperi, J.; Garnaud, S.; Rocher, V.; Moilleron, R. Priority pollutants in wastewater and combined sewer overflow. Science of Total Environment. 2008, 407(1), 263-272.

(40) Abduljabbar, H. Steroids: Clinical Aspect. BoD-Books on Demand. 2011.

(41) Bowers Jr, G.N.; McComb, R.B.; Christensen, R.; Schaffer, R.J. High-purity 4-nitrophenol: purification, characterization, and specifications for use as a spectrophotometric reference material. Clinical Chemistry. 1980, 26(6), 724-729.

(42) Ravindra, K.; Sokhi, R.; Van Grieken, R. Atmospheric polycyclic aromatic hydrocarbons: source attribution, emission factors and regulation. Atmospheric Environment. 2008, 42(13), 2895-2921.

(43) Johnsen, A.R.; Wick, L.Y.; Harms, H.J. Principles of microbial PAH-degradation in soil. Environmental Pollution. 2005, 133(1), 71-84.

(44) Yan, Z.; Yang, H.; Dong, H.; Ma, B.; Sun, H.; Pan, T.; Jiang, R.; Zhou, R.; Shen, J.; Liu, J. Occurrence and ecological risk assessment of organic micropollutants in the lower reaches of the Yangtze River, China: A case study of water diversion. Environmental Pollution. 2018, 239, 223-232.

(45) Richardson, S.D.; Ternes, T.A. Water analysis: emerging contaminants and current issues. Analytical chemistry. 2011, 83(12), 4614-4648.

(46) Richardson, S.D.; Kimura, S.Y. Water analysis: emerging contaminants and current issues. Analytical chemistry. 2016, 88(1), 546-582.

(47) Terechovs, A.K.; Ansari, A.J.; McDonald, J.A.; Khan, S.J.; Hai, F.I.; Knott, N.A.; Zhou, J.; Nghiem, L.D. Occurrence and bioconcentration of micropollutants in Silver Perch (Bidyanus bidyanus) in a 
reclaimed water reservoir. Science of Total Environment. 2019, 650, 585-593.

(48) Vos, J.G.; Dybing, E.; Greim, H.A.; Ladefoged, O.; Lambré, C.; Tarazona, J.V.; Brandt, I.; Vethaak, A.D. Health effects of endocrine-disrupting chemicals on wildlife, with special reference to the European situation. Critical Reviews in Toxicology. 2000, 30(1), 71-133.

(49) Richardson, S.D.; Ternes, T.A. Water analysis: emerging contaminants and current issues. Analytical chemistry. 2014, 86(6), 2813-2848.

(50) Zeng, F.; Cui, K.; Xie, Z.; Wu, L.; Liu, M.; Sun, G.; Lin, Y.; Luo, D.; Zeng, Z.J. Phthalate esters (PAEs): emerging organic contaminants in agricultural soils in peri-urban areas around Guangzhou, China. Environmental Pollution. 2018, 156(2), 425-434.

(51) Jos, A.; Repetto, G.; Ríos, J.C.; del Peso, A.; Salguero, M.; Hazen, M.J.; Molero, M.L.; FernándezFreire, P.; Pérez-Martín, J.M.; Labrador, V. Ecotoxicological evaluation of the additive butylated hydroxyanisole using a battery with six model systems and eighteen endpoints. Aquatic Toxicology. 2005, 71(2), 183-192.

(52) Chau, H.T.C.; Kadokami, K.; Duong, H.T.; Kong, L.; Nguyen, T.T.; Nguyen, T.Q.; Ito, Y. Occurrence of 1153 organic micropollutants in the aquatic environment of Vietnam. Environmental Science and Pollution Research. 2018, 25(8), 7147-7156.

(53) Carlsson, C.; Johansson, A. K.; Alvan, G.; Bergman, K.; Kühler, T. Are pharmaceuticals potent environmental pollutants?: Part I: Environmental risk assessments of selected active pharmaceutical ingredients. Science of Total Environment. 2006, 364(1-3), 67-87.

(54) Schwab, B.W.; Hayes, E.P.; Fiori, J.M.; Mastrocco, F.J.; Roden, N.M.; Cragin, D.; Meyerhoff, R.D.; Vincent, J.; Anderson, P.D. Human pharmaceuticals in US surface waters: a human health risk assessment. Regulatory Toxicology and Pharmacology. 2005, 42(3), 296-312.

(55) Perazzolo, C.; Morasch, B.; Kohn, T.; Smagnet, A.; Thonney, D.; Chèvre, N. Occurrence and fate of micropollutants in the Vidy Bay of Lake Geneva, Switzerland. Part I: Priority list for environmental risk assessment of pharmaceuticals. Environmental Toxicology and Chemistry. 2010, 29(8), 1649-1657.

(56) Verlicchi, P.; Al Aukidy, M.; Zambello, E. Occurrence of pharmaceutical compounds in urban wastewater: removal, mass load and environmental risk after a secondary treatment-A review. Science of the Total Environment. 2012, 429, 123-155.

(57) Ferguson, P.J.; Bernot, M.J.; Doll, J.C.; Lauer, T.E. Detection of pharmaceuticals and personal care products (PPCPs) in near-shore habitats of southern Lake Michigan. Science of Total Environment. 2013, 458, 187-196.

(58) Munz, N.A.; Burdon, F.J.; De Zwart, D.; Junghans, M.; Melo, L.; Reyes, M.; Schönenberger, U.; Singer, H.P.; Spycher, B.; Hollender, J. Pesticides drive risk of micropollutants in wastewater-impacted streams during low flow conditions. Water Research. 2017, 110, 366-377.

(59) Leung, H.W.; Jin, L.; Wei, S.; Tsui, M.M.P.; Zhou, B.; Jiao, L.; Cheung, P.C.; Chun, Y.K.; Murphy, M.B.; Lam, P.K. Pharmaceuticals in tap water: human health risk assessment and proposed monitoring framework in China. Environmental Health Perspectives. 2013, 121(7), 839-846.

(60) Al Aukidy, M.; Verlicchi, P.; Voulvoulis, N.J. A framework for the assessment of the environmental risk posed by pharmaceuticals originating from hospital effluents. Science of Total Environment. 2014, 493, 
54-64.

(61) Díaz-Garduño, B.; Pintado-Herrera, M.; Biel-Maeso, M.; Rueda-Márquez, J.; Lara-Martín, P.; Perales, J.; Manzano, M.; Garrido-Pérez, C.; Martín-Díaz, M. Environmental risk assessment of effluents as a whole emerging contaminant: Efficiency of alternative tertiary treatments for wastewater depuration. Water Research. 2017, 119, 136-149.

(62) Sodré, F.F.; Dutra, P.M.; dos Santos, V.P. Pharmaceuticals and personal care products as emerging micropollutants in Brazilian surface waters: a preliminary snapshot on environmental contamination and risks. Eclética Química Journal. 2018, 43, 22-34.

(63) Loos, R.; Gawlik, B.M.; Locoro, G.; Rimaviciute, E.; Contini, S.; Bidoglio, G. EU-wide survey of polar organic persistent pollutants in European river waters. Environmental Pollution. 2009, 157(2), 561568.

(64) Yang, Y. Y.; Toor, G.S.; Wilson, P.C.; Williams, C.F. Micropollutants in groundwater from septic systems: Transformations, transport mechanisms, and human health risk assessment. Water Research. 2017, 123, 258-267.

(65) Stasinakis, A.S.; Mermigka, S.; Samaras, V.G.; Farmaki, E.; Thomaidis, N.S. Occurrence of endocrine disrupters and selected pharmaceuticals in Aisonas River (Greece) and environmental risk assessment using hazard indexes. Environmental Science and Pollution Research. 2012, 19(5), 1574-1583.

(66) Tovar-Sánchez, A.; Sánchez-Quiles, D.; Basterretxea, G.; Benedé, J.L.; Chisvert, A.; Salvador, A.; Moreno-Garrido, I.; Blasco, J. Sunscreen products as emerging pollutants to coastal waters. Plos One. 2013, $8(6)$, e65451.

(67) USEPA. Intergrated Risk Information System. 2010.

(68) WHO. Pesticide residues in food: toxicological evaluations. 2002, 443.

(69) Teuschler, L.K.; Dourson, M.L.; Stiteler, W.M.; McClure, P.; Tully, H. Health risk above the reference dose for multiple chemicals. Regulatory Toxicology and Pharmacology. 1999, 30(2), S19-S26.

(70) Snyder, S.A. Occurrence, treatment, and toxicological relevance of EDCs and pharmaceuticals in water. Ozone: Science \& Engineering. 2008, 30(1), 65-69.

(71) Cho, E.; Khim, J.; Chung, S.; Seo, D.; Son, Y. Occurrence of micropollutants in four major rivers in Korea. Science of Total Environment. 2014, 491, 138-147.

(72) Ghekiere, A.; Verdonck, F.; Claessens, M.; Monteyne, E.; Roose, P.; Wille, K.; Goffin, A.; Rappé, K.; Janssen, C.R. Monitoring micropollutants in marine waters, can quality standards be met? Marine Pollution Bulletin. 2013, 69(1-2), 243-250.

(73) Li, X.; Shang, X.; Luo, T.; Du, X.; Wang, Y.; Xie, Q.; Matsuura, N.; Chen, J.; Kadokami, K. Screening and health risk of organic micropollutants in rural groundwater of Liaodong Peninsula, China. Environmental Pollution. 2016, 218, 739-748.

(74) Munz, N.A.; Fu, Q.; Stamm, C.; Hollender, J. Internal concentrations in gammarids reveal increased risk of organic micropollutants in wastewater-impacted streams. Environmental Science \& Technology. 2018, 52(18), 10347-10358.

(75) Kuzmanović, M.; Ginebreda, A.; Petrović, M.; Barceló, D. Risk assessment based prioritization of 200 organic micropollutants in 4 Iberian rivers. Science of Total Environment. 2015, 503, 289-299. 
(76) Fromme, H.; Küchler, T.; Otto, T.; Pilz, K.; Müller, J.; Wenzel, A. Occurrence of phthalates and bisphenol A and F in the environment. Water Research. 2002, 36(6), 1429-1438. 